\title{
Mixture modeling of microarray gene expression data
} Yang Yang*1, Adam P Tashman' ${ }^{1}$, Jung Yeon Lee ${ }^{1}$, Seungtai Yoon ${ }^{2}$, Wenyang Mao ${ }^{1}$, Kwangmi Ahn ${ }^{3}$, Wonkuk Kim ${ }^{1}$, Nancy R Mendell ${ }^{1}$, Derek Gordon ${ }^{4}$ and Stephen J Finch ${ }^{1}$

\begin{abstract}
Address: ${ }^{1}$ Department of Applied Mathematics and Statistics, Stony Brook University, Stony Brook, New York 11790, USA, ${ }^{2}$ Cold Spring Harbor Laboratory, Cold Spring Harbor, New York 11724, USA, ${ }^{3}$ Department of Health Evaluation Sciences, A210, Penn State College of Medicine, 600 Centerview Drive, Hershey, Pennsylvania 17033, USA and ${ }^{4}$ Department of Genetics, Rutgers University, 145 Bevier Road, Room 128, Piscataway, New Jersey 08854, USA

Email: Yang Yang* - yayang@ams.sunysb.edu; Adam P Tashman - apt4c@hotmail.com; Jung Yeon Lee - yjysuny@ams.sunysb.edu; Seungtai Yoon - yoon@cshl.edu; Wenyang Mao - wenyangmao@hotmail.com; Kwangmi Ahn - kxa14@psu.edu;

Wonkuk Kim - wkim@ams.sunysb.edu; Nancy R Mendell - nancy.mendell@stonybrook.edu; Derek Gordon - gordon@biology.rutgers.edu; Stephen J Finch - stephen.finch@stonybrook.edu

* Corresponding author
\end{abstract}

from Genetic Analysis Workshop 15

St. Pete Beach, Florida, USA. II-15 November 2006

Published: 18 December 2007

BMC Proceedings 2007, I (Suppl I):S50

This article is available from: http://www.biomedcentral.com/I753-656I/I/SI/S50

C 2007 Yang et al; licensee BioMed Central Ltd.

This is an open access article distributed under the terms of the Creative Commons Attribution License (http://creativecommons.org/licenses/by/2.0),

which permits unrestricted use, distribution, and reproduction in any medium, provided the original work is properly cited.

\begin{abstract}
About $28 \%$ of genes appear to have an expression pattern that follows a mixture distribution. We use first- and second-order partial correlation coefficients to identify trios and quartets of non-sexlinked genes that are highly associated and that are also mixtures. We identified 18 trio and 35 quartet mixtures and evaluated their mixture distribution concordance. Concordance was defined as the proportion of observations that simultaneously fall in the component with the higher mean or simultaneously in the component with the lower mean based on their Bayesian posterior probabilities. These trios and quartets have a concordance rate greater than $80 \%$. There are 33 genes involved in these trios and quartets. A factor analysis with varimax rotation identifies three gene groups based on their factor loadings. One group of 18 genes has a concordance rate of $56.7 \%$, another group of 8 genes has a concordance rate of $60.8 \%$, and a third group of 7 genes has a concordance rate of $69.6 \%$. Each of these rates is highly significant, suggesting that there may be strong biological underpinnings for the mixture mechanisms of these genes. Bayesian factor screening confirms this hypothesis by identifying six single-nucleotide polymorphisms that are significantly associated with the expression phenotypes of the five most concordant genes in the first group.
\end{abstract}




\section{Background}

McLachlan et al. [1] introduced a mixture analysis approach to the clustering of microarray expression data, in particular, of tissue samples on a very large number of genes. Maclean et al. [2] developed the SKUMIX algorithm, which can test whether a mixture model fits the genetic data with skewness removed by Box-Cox transformation [3], and then used a likelihood-ratio test (LRT) statistic to determine whether the two-component model appears to fit the data better than the single-component model. Given the high degree of correlation among the gene expression variables, Simon's work [4] suggests that one use first- and second-order partial correlation coefficients to find trios and quartets of genes that have high degrees of "explanation." Here we focus on trios and quartets comprising only non-sex-linked genes that appear to follow a mixture distribution to explore the associations of these mixing mechanisms. For example, if there is one common mixture mechanism governing all of the genes in a set, then the fraction of subjects simultaneously falling in the same mixing component of these genes would be high. We then use varimax factor anlaysis $[5,6]$ to see whether we can identify more than four genes operating under a common mixing mechanism. One confirmation that the common mixture mechanism has biological importance would be to identify genetic relationships between a subject's single-nucleotide polymorphism (SNP) genotypes and expression phenotypes. Bayesian factor screening (BFS) [7] is one statistical strategy proposed to identify these relations. In this paper, the mixture model-based approach with extended SKUMIX algorithm, partial correlation analysis, factor analysis, and BFS are systematically combined to analyze the Problem 1 data set in Genetic Analysis Workshop 15 (GAW15) [8,9].

\section{Methods}

\section{Box-Cox family of transformations}

Given the expression intensities $x_{1, j}, x_{2, j}, \ldots, x_{n, j}(\mathrm{n}=194)$ for the $j^{\text {th }}(j=1,2, \ldots, 3554)$ gene, the Box-Cox family [3] transforming $x_{j}=\left(\begin{array}{llllll}x_{1}, j, & x_{2}, & j, \ldots, & x_{n,}\end{array}\right)$ to $x_{j}^{\left(p_{j}\right)}=\left(x_{1, j}^{\left(p_{j}\right)}, x_{2, j}^{\left(p_{j}\right)}, \ldots, x_{n, j}^{\left(p_{j}\right)}\right)$ with power parameter $p_{j}$ is:

$$
x_{j}^{\left(p_{j}\right)}=\left\{\begin{array}{ll}
\left(x_{j}^{p_{j}}-1\right) / p_{j}, & p_{j} \neq 0 \\
\ln \left(x_{j}\right), & p_{j}=0
\end{array} .\right.
$$

The expression intensities transformed here are the original observations rather than the $\log _{2}$ values reported in the data set. The 0.3-power transformation is the transformation that maximizes the probability plot correlation coefficient (PPC, see Filliben [10]) for the greatest number of genes.

\section{Mixture analysis using Gaussian mixture model}

The SKUMIX algorithm is extended in our mixture analysis. First, we applied the Box-Cox family of power transformations without the scale parameter (see Eq. (1)). Second, we considered a wider interval $[0,1.5]$ than the one recommended by Maclean et al. [2] for selecting the optimal power parameter. Third, as suggested by Ning et al. [11], we used 6.9 as the 0.05 critical value for LRT of "a single component distribution" vs. "a mixture distribution of two components."

\section{Partial correlation analysis}

We calculate the Pearson product moment correlation coefficients $r_{i j}=r\left(x_{i}, x_{j}\right)$, first-order partial correlations $r_{i j . k}$ $=r\left(x_{i}, x_{j} \mid x_{k}\right)$ and second-order partial correlation coefficients $r_{i j . k l}=r\left(x_{i}, x_{j} \mid x_{k^{\prime}} x_{l}\right)$ [12] for expression phenotype variables whose values are the 0.3 -power Box-Cox transformed expressions. The partial correlation criteria are:

$$
\begin{gathered}
\mathbf{T}:\left|r\left(x_{i}, x_{j}\right)\right|>0.8 \text { and } r_{i j}^{2}-r_{i j . k}^{2}>0.63 \text { for } i \neq j \neq k \\
\mathrm{Q}:\left|r_{i j}\right|>0.8, r_{i j}^{2}-r_{i j, k l}^{2}>0.63, r_{i j}^{2}-r_{i j, k}^{2}<0.63 \text {, and } r_{i j}^{2}-r_{i j, l}^{2}<0.63 \text { for } i \neq j \neq k \neq l .
\end{gathered}
$$

The last two inequalities in criterion $Q$ reduce redundancy by removing quartets built on trios. We identify trios of expression phenotype variables $\left(x_{i}, x_{j}, x_{k}\right)$ that meet criterion T and quartets $\left(x_{i^{\prime}}, x_{j^{\prime}}, x_{k^{\prime}}, x_{l}\right)$ that meet criterion Q.

\section{Measure of common mixing mechanism}

When a gene expression variable appeared to be a mixture, we fit a mixture of two Gaussian components with equal variance using MCLUST [13] and classified each subject into the component with the largest Bayesian posterior probability [14]. We called the component with estimated probability less than 0.5 the "uncommon component" and the other one the "common component." The concordance rate $(C)$ in a gene set is the ratio of subjects that simultaneously fall into the uncommon or the common components for all the genes in the set. A value of $C$ close to 1 suggests a common mixture mechanism. We selected genes in a trio or quartet with $C \geq 80 \%$ for the factor analysis. Fleiss' statistic $\kappa[15]$ was used to assess agreement. A value of $\kappa>0.75$ indicated excellent agreement, while $\kappa<0.40$ indicated poor agreement [16].

\section{Factor analysis}

Each gene expression variable that appeared to be a mixture and was present in one or more trios or quartets was included in a factor analysis using varimax rotation.

\section{Bayesian factor screening}

We used BFS $[7,17]$ to identify SNPs significantly associated with expressions of the genes from the factor analysis. 
We only considered the regression model with secondorder interactions:

$$
y=\alpha+\sum_{j=1}^{S} \beta_{j} x_{j}+\sum_{i<j} \beta_{i j} x_{i} x_{j}+\varepsilon,
$$

where the values of $x_{1}, x_{2}, \ldots, x_{S}$ are recoded genotypes ( 1 for minor homozygotes, 2 for heterozygotes, 3 for major homozygotes, and -2 for missing data) of $S$ (2682) consistent and informative SNPs that may have linear main effects and/or interaction effects on the gene expression variable $\gamma$. Let $\gamma$ be the indicator vector such that $\gamma_{j}=0$ if $\beta_{j}$ $=0$ and $\beta_{i j}=0$ for all $i \neq j$, and $\gamma_{j}=1$ if otherwise. Then a model (or an element) in the model space can be represented by a binary vector $\gamma=\left(\gamma_{1}, \gamma_{2}, \ldots, \gamma_{S}\right)$ that ranges from $\gamma^{(1)}=(0,0, \ldots, 0)$ to $\gamma^{\left(2^{\mathrm{S}}\right)}=(1,1, \ldots, 1)$, with the model size defined as $m=\sum_{j=1}^{S} \gamma_{j} \in[0, S]$. In our study, we set the model size $m=6$, the chain length $C L=200,000$, and the magnitude of the effect relative to the experimental noise $\lambda=1.5$. We use the Java program developed by Yoon [17] to find the optimal model from the model subspace consisting of $C_{6}^{2,682}=5.14 \times 10^{17}$ elements. The output gives an estimate of each SNP's marginal posterior probability (MPP) of appearing in the 200,000 selected models. An MPP close to 1 suggests that the SNP is an important factor (either as a main effect or as one of two terms in an interaction) for the gene expression variable.

\section{Results}

Of the 3554 gene expressions analyzed, 2561 appear to follow a normal distribution. After a Box-Cox transformation to maximize the PPC, 659 give evidence of being a mixture with two components, and 334 appear to have three components. Figure 1 contains the histogram of the 0.3-power Box-Cox transformed expressions of TUBG1 that appears to follow a mixture of two components. The left component is the uncommon one, with estimated proportion $15.7 \%$ and estimated mean of 19.9 . The right component is the common one, with estimated proportion $84.3 \%$ and estimated mean of 26.1 .

We find 233 trios containing 54 genes that meet criterion $\mathrm{T}$, and 115,840 quartets containing 3554 genes that meet criterion Q. Of the 233 trios, 88 include only mixture distributions (involving a total of 29 genes). Of the 115,840 quartets, 7342 include only mixture distributions (involving a total of 902 genes). A number of trios and quartets contain only sex-linked genes. When we exclude these sexlinked gene sets, there are 18 trios and 35 quartets with a

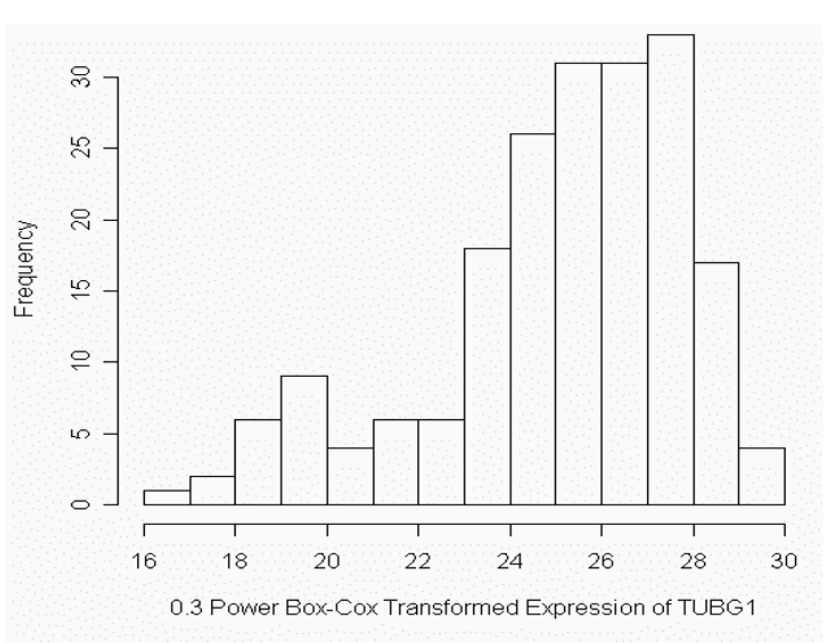

Figure I

Histogram of the 0.3-power Box-Cox transformed TUBGI.

value of $C \geq 80 \%$. These trios and quartets contain 33 non-sex-linked genes in total.

One example is the quartet containing HNRPAB, PSMD2, TUBG1, and AHSA1. Each of these genes appears to be a mixture with very small $p$-value; Figure 1 is the histogram of the 0.3-power transformed expressions of TUBG1. The correlation between PSMD2 and HNRPAB (using the 0.3power transformed expressions) is 0.825 , and the partial correlation between PSMD2 and HNRPAB controlling for TUBG 1 and AHSA 1 is 0.094 . Table 1 is the four-way contingency table in which each subject is classified by the Bayesian posterior probability into the common or uncommon component. The $C$ value for this set of genes is $83.50 \%$. Specifically, 136 of 194 subjects are simultaneously common and 26 are simultaneously uncommon in these four genes so that 162 of 194 subjects (that is, $83.50 \%)$ are concordant. There are, respectively, 14 $(1+6+7)$ and $9(6+3)$ additional subjects that fall into the common and uncommon components of three genes out of the four, suggesting a larger concordance rate for smaller gene sets.

A factor analysis on the 0.3-power transformed gene expression levels of the 33 non-sex-linked genes identifies three factor groups. As listed in Table 2, Factor 1 appears to consist of 18 genes, Factor 2 appears to consist of 8 genes, and Factor 3 appears to consist of 7 genes. A trio with a high value of $C$ contains genes from Factor 2 or from Factor 3. A quartet with a high value of $C$ contained all genes either from Factor 1 or from Factor 3.

We then examined whether the genes in each factor group follow a common mixture mechanism. In each factor group, we started with the pair of genes that have the high- 
Table I: Contingency table for TUBGI, AHSAI, PSMD2, and HNRPAB

\begin{tabular}{|c|c|c|c|c|c|c|}
\hline \multicolumn{7}{|c|}{ Subjects Classified into Common or Uncommon Components } \\
\hline \multirow[t]{2}{*}{ TUBGI } & \multirow[t]{2}{*}{$A H S A I \downarrow$} & \multirow{2}{*}{$\begin{array}{l}\text { PSMD2 } \rightarrow \\
\text { HNRPAB } \rightarrow\end{array}$} & \multicolumn{2}{|c|}{ Common } & \multicolumn{2}{|c|}{ Uncommon } \\
\hline & & & Common & Uncommon & Common & Uncommon \\
\hline \multirow[t]{2}{*}{ Common } & Common & & 136 & 6 & 7 & 4 \\
\hline & Uncommon & & 1 & 3 & 2 & 6 \\
\hline \multirow[t]{2}{*}{ Uncommon } & Common & & 0 & 0 & 0 & 3 \\
\hline & Uncommon & & 0 & 0 & 0 & 26 \\
\hline
\end{tabular}

est $C$ value and added the gene from the factor group that least reduces $C$. For example, the first two genes $A_{1}=$ \{AHSA1, ELAC2 \} have the largest $C=94.85 \%$, with $\kappa=$ 0.8257 . The gene CCT3 had the least reduction in $C$ value of the 16 genes remaining in Factor 1. We include genes from Factor 1 sequentially until we got the final gene group $A_{17}=A_{16} \cup\{$ SOD 1$\}$ with $C=56.70 \%$ and $\kappa=$ 0.6440 . For this factor group, the reduction in $C$ value with adding one gene to the set ranges from $1 \%$ to $3 \%$. Similar results hold for Factor groups 2 and 3.

We extended the mixture analysis with BFS applied to the five most concordant genes in Group 1 (AHSA1, ELAC2, CCT3, TUBG1, and TACC3, with $C>85 \%$ and $\kappa>0.75$ ). For each of these genes, BFS identifies six SNPs that have very large MPPs, as shown in Table 3.

\section{Conclusion}

About 28\% of genes from GAW15 Problem 1 appear to follow a two- or threecomponent mixture distribution. Important structural relations seem to be partially disentangled using first- and second-order partial correlation matrices. These partial correlation coefficients can be effectively used to identify trios and quartets of genes that have a more complex structure. There are 18 trios and 35 quartets in which the genes are all non-sex-linked but follow a common mixture distribution with $C \geq 80 \%$. That is, the underlying mixture mechanisms of these genes appear to be highly associated. This pattern of association appears to involve a large number of genes. A computational strategy using the varimax rotation in a factor analysis finds a group of 18 genes with $C=56.7 \%$, another group of 9 genes with $C=60.8 \%$, and a third group of 7 genes with $C=69.6 \%$. The R package MIXMECH that has been developed here for

Table 2: Concordance rate $(C)$ for sets of genes selected from factors

\begin{tabular}{|c|c|c|c|c|c|c|c|c|c|}
\hline \multirow[b]{2}{*}{ Seq } & \multicolumn{3}{|c|}{ Factor I } & \multicolumn{3}{|c|}{ Factor 2} & \multicolumn{3}{|c|}{ Factor 3} \\
\hline & Gene & $C(\%)$ & $\kappa$ & Gene & $C(\%)$ & $\kappa$ & Gene & $C(\%)$ & $\kappa$ \\
\hline 1 & $\begin{array}{l}\text { AHSAI, } \\
\text { ELAC2 }\end{array}$ & 94.85 & 0.8257 & $\begin{array}{l}\text { RPL32, } \\
\text { RPSI8 }\end{array}$ & 92.78 & 0.8278 & $\begin{array}{c}\text { PRKARIA, } \\
\text { STI } 3\end{array}$ & 93.30 & 0.8609 \\
\hline 2 & ССТ3 & 90.72 & 0.7773 & RPSI 5 & 87.63 & 0.8104 & MATR3 & 86.08 & 0.8117 \\
\hline 3 & TUBG I & 88.66 & 0.7663 & RPS28 & 81.96 & 0.7701 & PPMIB & 80.93 & 0.7934 \\
\hline 4 & TACC3 & 85.57 & 0.7555 & RPSI0a & 76.29 & 0.7081 & PDCDIO & 77.84 & 0.7825 \\
\hline 5 & NDUFS6 & 82.99 & 0.7495 & RPSI9 & 72.68 & 0.6677 & SF3BI & 73.7I & 0.7684 \\
\hline 6 & CDC45L & 80.41 & 0.7273 & B2M & 68.56 & 0.6340 & G3BP2 & 69.59 & 0.7521 \\
\hline 7 & DHX9 & 77.84 & 0.7216 & RPSI0b & 64.43 & 0.5578 & NA & NA & NA \\
\hline 8 & FENI & 75.26 & 0.7096 & PABPCI & 60.82 & 0.5510 & NA & NA & NA \\
\hline 9 & HNRPAB & 73.7I & 0.7070 & NA & NA & NA & NA & NA & NA \\
\hline 10 & PSMD2 & 72.16 & 0.7034 & NA & NA & NA & NA & NA & NA \\
\hline 11 & CSEIL & 70.10 & 0.6931 & NA & NA & NA & NA & NA & NA \\
\hline 12 & C20orf24 & 67.53 & 0.6800 & NA & NA & NA & NA & NA & NA \\
\hline 13 & JTVI & 65.46 & 0.6755 & NA & NA & NA & NA & NA & NA \\
\hline 14 & LANCL2 & 63.92 & 0.6662 & NA & NA & NA & NA & NA & NA \\
\hline 15 & TSTA3 & 62.37 & 0.6572 & NA & NA & NA & NA & NA & NA \\
\hline 16 & ССТ7 & 59.79 & 0.6514 & NA & NA & NA & NA & NA & NA \\
\hline 17 & SODI & 56.70 & 0.6440 & NA & NA & NA & NA & NA & NA \\
\hline
\end{tabular}

aMeasured on probe set 200095 _ _ at

bMeasured on probe set 200817_x_at 
Table 3: Marginal posterior probabilities (MPPs) of six SNPs associated with AHSAI, ELAC2, CCT3, TUBGI, and TACC3

\begin{tabular}{|c|c|c|c|c|c|}
\hline SNP/Location & AHSAI & ELAC2 & CCT3a & TUBGI & TACC3 \\
\hline rs|438676/chr 5 & 0.9976 & 0.9976 & 0.3024 & 0.5618 & 0.9976 \\
\hline rs|560|43/chr 5 & 0.9976 & 0.9976 & 0.3024 & 0.5618 & 0.9976 \\
\hline rs|453389/chr II & 0.9976 & 0.9976 & 0.3024 & 0.5618 & 0.9976 \\
\hline rs|945465/chr II & 0.9976 & 0.9976 & 0.3024 & 0.5618 & 0.9976 \\
\hline rsl993205/chr II & 0.9976 & 0.9976 & 0.3024 & 0.5618 & 0.9976 \\
\hline rs204304I/chr 18 & 0.9992 & 0.9992 & 0.9992 & 0.5633 & 0.9992 \\
\hline
\end{tabular}

aThere are five SNPs not reported here with MPP between 0.3024 and 0.9992 . These five SNPs do not have MPPs higher than the ones reported for genes AHSAI, ELAC2, TUBGI, and TACC3.

the mixture analysis of microarray expression data is freely available at the websites http://www.ams.sunysb.edu/ yayang and http://www.sinc.sunysb.edu/Stu/atashman.

The significance of these findings is not immediately clear. For example, one possible source of a mixture mechanism that is not substantively interesting is the non-homogenous measurement process of the gene expressions. The data used here are from 14 pedigrees rather than from a random sample of cases or controls. Therefore, we do not know the magnitude of the effect of dependence among subjects generated by the family structure. In results not shown here, however, we obtained results parallel to these when we restricted our analysis to the 56 unrelated founders, suggesting that the effect of the intra-familial dependence is minor. As always, replication of these results on an independent data set is a crucial step to confirm the scientific value of this approach and our findings.

Nevertheless, the high concordance rates and high Fleiss $\kappa$ coefficients suggest that there may be a common mechanism determining which component a subject falls into. More importantly, the BFS result showing a strong association between the five most concordant genes in Group 1 with the six SNPs strongly suggests that there is an underlying biological mechanism.

\section{Competing interests}

The author(s) declare that they have no competing interests.

\section{Acknowledgements}

The data were collected under HG002386. Our work is supported by GAW grant ROI GM3 I575. NRM's research is supported in part by NIMH ROI MH07I523, and SJF's is supported in part by NIMH 2ROI

MH04480II4AI. We thank our group members (GAWI5 Group 5), the reviewers, the editors, Cathy Falk and Deborah L. Levy for their thoughtful comments.

This article has been published as part of BMC Proceedings Volume I Supplement I, 2007: Genetic Analysis Workshop 15: Gene Expression Analysis and Approaches to Detecting Multiple Functional Loci. The full contents of the supplement are available online at http://www.biomedcentral.com/ $\mid 753-6561 /$ l issue $=$ SI.

\section{References}

I. McLachlan GJ, Bean RW, Peel D: A mixture model-based approach to the clustering of microarray expression data. Bioinformatics 2002, 18:4 |3-422.

2. Maclean CJ, Morton NE, Elston RC: Skewness in commingled distributions. Biometrics 1976, 32:695-699.

3. Box GEP, Cox DR: An analysis of transformations. J R Stat Soc Ser B 1964, 26:2 I I-246.

4. Simon HA: Spurious correlation: a causal interpretation. J $A m$ Stat Assoc 1954, 49:467-479.

5. Gorsuch RL: Factor Analysis Hillsdale, NJ: Lawrence Erlbaum; 1983.

6. McLachlan GF, Peel D, Bean RW: Modelling high dimensional data by mixtures of factor analyzers. Comput Stat Data Anal 2003, 4l:379-388.

7. Yoon S, Suh YJ, Mendell NR, Ye KQ: A Bayesian approach for applying Haseman-Elston methods. BMC Genetics 2005, 6(SuppI I):S39.

8. Morley M, Molony CM, Weber TM, Devlin JL, Ewens KG, Spielman RS, Cheung VG: Genetic analysis of genome-wide variation in human gene expression. Nature 2004, 430:743-747.

9. Cheung VG, Spielman RS, Ewens KG, Weber TM, Morley M, Burdick $\mathrm{JT}$ : Mapping determinants of human gene expression by regional and genome-wide association. Nature 2005, 437: $1365-1369$

10. Filliben J: The probability plot correlation coefficient test for normality. Technometrics 1975, I7:III-II7.

II. Ning YM, Finch SJ: The null distribution of the likelihood ratio test for a mixture of two normals after a restricted Box-Cox transformation. Comm Stat Simul Comp 2000, 29:449-46I.

12. Sokal RR, Rohlf FJ: Biometry New York: Freeman; 1995.

13. Fraley C, Raftery AE: Model-based clustering, discriminant analysis, and density estimation. J Am Stat Assoc 2002, 97:6I|-63|.

14. Casella G, Berger RL: Statistical Inference Belmont, CA: Wadsworth \& Brooks/Cole; 1990.

15. Fleiss JL: Measuring nominal scale agreement among many raters. Psychol Bull 1971, 76:378-382.

16. Fleiss JL: Statistical Methods for Rates and Proportions New York: John Wiley \& Sons; 1981.

17. Yoon S: Bayesian factor screening. In Dissertation Stony Brook University, Department of Applied Mathematics and Statistics; 2006.

Publish with Biomed Central and every scientist can read your work free of charge

"BioMed Central will be the most significant development for disseminating the results of biomedical research in our lifetime. "

Sir Paul Nurse, Cancer Research UK

Your research papers will be:

- available free of charge to the entire biomedical community

- peer reviewed and published immediately upon acceptance

- cited in PubMed and archived on PubMed Central

- yours - you keep the copyright 